\title{
Endurance Exercise Training-Attenuated Diabetic Kidney Disease with Muscle Weakness in Spontaneously Diabetic Torii Fatty Rats
}

\author{
Hitoshi Kotake $^{a}$ Shohei Yamada ${ }^{a}$ Yuji Ogurab Shiika Watanabe ${ }^{a}$ \\ Kazuho Inoue $^{c}$ Daisuke Ichikawa ${ }^{a}$ Takeshi Sugaya $^{a} K_{\text {Keiichi Ohata }}{ }^{a}$ \\ Yasunori Natsuki $^{d}$ Seiko Hoshino ${ }^{c}$ Minoru Watanabe ${ }^{e}$ Kenjiro Kimura ${ }^{f}$ \\ Yugo Shibagaki ${ }^{a}$ Atsuko Kamijo-Ikemori ${ }^{a, c, e}$

\begin{abstract}
aDivision of Nephrology and Hypertension, Department of Internal Medicine, St. Marianna University School of Medicine, Kawasaki, Japan; 'Department of Physiology, St. Marianna University School of Medicine, Kawasaki,

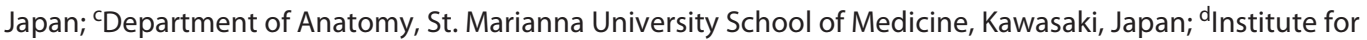
Ultrastructural Morphology, St. Marianna University School of Medicine, Kawasaki, Japan; elnstitute for Animal Experimentation, St. Marianna University Graduate School of Medicine, Kawasaki, Japan; fJCHO Tokyo Takanawa Hospital, Tokyo, Japan
\end{abstract}

\section{Keywords}

Type 2 diabetes · Diabetic kidney disease · Exercise · Endurance exercise training - Spontaneously diabetic Torii fatty rat

\footnotetext{
Abstract

Background: The aim of this study was to evaluate protective effects of endurance exercise training against diabetic kidney disease (DKD) with muscle weakness by using male spontaneously diabetic Torii (SDT) fatty rats as type 2 diabetic animal models with obesity, hypertension, and hyperlipidemia. Methods: Eight-week-old SDT fatty rats $(n=12)$ and Sprague-Dawley (SD) rats $(n=10)$ were randomly divided into exercise (Ex; SDT-Ex: $n=6$, SD-Ex: $n=5)$ and sedentary groups (SDT-Cont: $n=6$, SD-Cont: $n=5$ ), respectively. Each group underwent regular treadmill exercise 4 times a week from ages 8-16 weeks. Results: The exercise attenuated hypertension and hyperlipidemia and prevented increases in
}

renal parameter levels without affecting blood glucose levels. In the SDT fatty rats, it prevented induction of renal morphological abnormalities in the interstitium of the superficial and intermediate layers of the cortex. Downregulated expression of endothelial nitric oxide synthase in the glomerulus of the SDT fatty rats was significantly upregulated by the exercise. The exercise upregulated the renal expressions of both medium-chain acyl-CoA dehydrogenase and peroxisome proliferator-activated receptor $\gamma$ coactivator-1 1 related to fatty acid metabolism. It increased muscle strength and both muscle weight and cross-sectional area of type Ilb muscle fibers in the extensor digitorum longus muscle in the SDT fatty rats. Conclusion: Endurance exercise training in type 2 diabetes ameliorates DKD by improving endothelial abnormality and enhancing fatty acid metabolism in addition to attenuated hypertension, hyperlipidemia, and muscle weakness independently of blood glucose levels.

(c) 2021 The Author(s)

Published by S. Karger AG, Basel karger@karger.com www.karger.com/kbr

Karger $\stackrel{\text { ! }}{=}$

BOPEN ACCESS
(C) 2021 The Author(s)

Published by S. Karger AG, Basel

This is an Open Access article licensed under the Creative Common Attribution-NonCommercial-4.0 International License (CC BY-NC) (http://www.karger.com/Services/OpenAccessLicense), applicable to the online version of the article only. Usage and distribution for commercial purposes requires written permission.
Correspondence to:

Atsuko Kamijo-Ikemori, a2kamijo@marianna-u.ac.jp 


\section{Introduction}

Diabetic kidney disease (DKD) is caused by various factors such as obesity, hypertension, and hyperlipidemia in addition to hyperglycemia [1-3]. Different pharmacological treatments that target these factors ameliorate DKD progression. However, under an aging society with increased number of elderly type 2 diabetes patients with impaired metabolic, physical, and cognitive functions, pharmacological treatments could cause detrimental effects such as adverse drug reactions, hypoglycemic attack, hypotension, and falling [4-6]. Therefore, preventive measures against DKD progression are important rather than relying solely on chemical agents. This new strategy will ameliorate DKD by attaining favorable levels of activities of daily living and quality of life in elderly patients.

Exercise training is useful for preventing muscle weakness, which leads to maintenance of activities of daily living and quality of life. Patients with chronic kidney disease were previously advised to avoid exercise training without obvious scientific basis. However, recent evidence suggests that exercise training shows reno-protective effects in clinical and experimental studies [7-13]. Prior studies using type 2 diabetic animal models reported that the reno-protective mechanism of exercise implicated antioxidative and anti-inflammatory effects and improved renal hemodynamics [14-16]. These findings suggest that exercise training is useful for inhibiting DKD progression and muscle weakness as a nonpharmacological measure.

Spontaneously diabetic Torii (SDT) fatty (SDT.CgLepr ${ }^{\mathrm{fa}} / \mathrm{JttJ} \mathrm{cl}$ ) rats mimic the human pathophysiology of type 2 diabetes with obesity, hypertension, and hyperlipidemia [17]. In our previous study, SDT fatty rats showed symptoms of DKD and muscle weakness at 16 weeks of age $[18,19]$. Therefore, the aim of this study was to investigate the beneficial effects of endurance exercise training on the kidneys and muscle by using SDT fatty rats.

\section{Materials and Methods}

\section{Animals}

All the procedures that involved animals were performed in accordance with the ethical standards or practice of the St. Marianna University School of Medicine or the institution where the study was conducted (approval numbers: 1908009-C and 2008012). Male SDT fatty rats derived from a Sprague-Dawley (SD) colony were used as type 2 diabetes models. Five-week-old male SDT fatty $(n=12)$ and age-matched control SD rats $(n=10)$ were obtained from CLEA Japan (Tokyo, Japan) and allowed free access to usual laboratory chow and water.
Table 1. Intensity of treadmill exercise in each weeks old of rats

\begin{tabular}{lll}
\hline Age, weeks old & Speed, $\mathrm{m} / \mathrm{min}$ & Duration, $\min$ \\
\hline 8 & -10 & 10 \\
9 & $10-12$ & 20 \\
10 & $10-12$ & 30 \\
11 & $12-14$ & 30 \\
12 & $14-16$ & 30 \\
13 & $16-18$ & 30 \\
14 & $16-20$ & 30 \\
15 & $16-20$ & 30 \\
16 & $16-20$ & 30 \\
\hline
\end{tabular}

The SDT fatty $(n=12)$ and SD rats $(n=10)$ were randomly divided into exercise (Ex) (SDT-Ex: $n=6$, SD-Ex: $n=5)$ and sedentary groups (SDT-Cont: $n=6$, SD-Cont: $n=5)$ at age 8 weeks. The exercise groups of SDT fatty $(n=6)$ and SD rats $(n=5)$ underwent regular treadmill exercise on a motor-driven treadmill (KN-73 TREADMILL; Natume Seisakusho, Co., Ltd., Tokyo, Japan) in an animal room different from the breeding room in the same institute, 4 times a week from ages $8-16$ weeks as endurance exercise training. The exercise intensity was modified from that in a previous report [20](Table 1). After warm-up with slowly increasing speed from 5 to $10 \mathrm{~m} / \mathrm{min}$ for $5 \mathrm{~min}$, the prescribed exercise with a $3^{\circ}$ slope was started at $10 \mathrm{~m} / \mathrm{min}$ for $10 \mathrm{~min} /$ day, 4 times a week for the first week. From the second to the eighth week, the speed and duration of the prescribed exercise were gradually increased to $16-20 \mathrm{~m} / \mathrm{min}$ according to the tolerance of the rats for $30 \mathrm{~min}$ after the warm-up for $5 \mathrm{~min} 4$ times a week. To keep the same environment as that of the exercise groups, the control groups of the SDT fatty $(n=6)$ and SD rats $(n=$ 5 ) were also moved and put in the room equipped with the treadmill during the exercise. Body weight, blood glucose levels, systolic blood pressure (SBP), chow consumption, and muscle strength were measured in all the rats every 4 weeks from ages 8-16 weeks. Muscle strength was measured using the forelimb grip test with a grip strength meter (MK-380CM/FM; Muromachi Kikai, Co., Ltd., Tokyo, Japan). The average of 3 measurements of muscle strength per animal per time point was used for the comparative analysis [18].

For the experiments, 8-, 12-, and 16-week-old rats were individually housed overnight in metabolic cages with free access to tap water. Urine samples were collected for the measurement of urinary markers. Under inhalation, anesthesia using $2 \%$ isoflurane, serum and kidney and leg muscle tissue samples were collected after $16 \mathrm{~h}$ of starvation at age 16 weeks [18]. The extracted kidneys were weighed and cut into pieces for various downstream analyses. The extracted muscle specimens were sectioned into the soleus muscle as a slow muscle and the extensor digitorum longus (EDL) muscle as a fast muscle. After weighing, each muscle was frozen individually in liquid nitrogen and stored at $-80^{\circ} \mathrm{C}$ for further analyses. The muscle strengths and weights of all the rats were normalized to their body weight.

\section{Blood Pressure Measurement}

SBP was measured using a tail-cuff apparatus (Softron BP-98A; Softron, Tokyo, Japan) every 4 weeks from ages $8-16$ weeks. The averages of 3 SBP measurements per animal per time point were recorded [18]. 


\section{Serum and Urinary Biochemistry}

Serum cystatin $C$ is not influenced by change in muscle volume and accurately reflects renal function compared to serum creatinine [21]. Therefore, serum cystatin $C$ was used for evaluation of renal function in the present study. The levels of serum cystatin $C$ and insulin, urinary rat L-FABP, and rat albumin were measured using the same commercially available kits as those described in our previous study [19]. The serum total cholesterol and triglyceride levels were measured in the SRL clinical laboratory testing services (Tokyo, Japan) [19]. Serum interleukin-6 (IL-6) and brainderived neurotrophic factor (BDNF) were measured using each ELISA kit from R\&D Systems (Minneapolis, MN, USA). Urinary levels were reported as daily excretion.

\section{Renal Histological and Morphometric Analyses}

The midsection of each excised kidney was dissected in the minor axial direction. Both pieces were fixed in $10 \%$ buffered formalin or methyl Carnoy's solution (60\% methanol, $30 \%$ chloroform, and $10 \%$ glacial acetic acid) and embedded in paraffin [19]. Serial sections ( $3 \mu \mathrm{m}$ thick) were prepared for renal histological assessment, including periodic acid-Schiff (PAS) staining and immunohistochemistry. For each animal, glomerulosclerosis was evaluated in PAS-stained sections by grading the extent of sclerosis in each glomerulus at $\times 400$ magnification, as described previously [19]. For evaluation of glomerular hypertrophy defined as enlargement of the glomerulus area, the size of glomerulus area was also measured by using an image analyzer at $\times 400$ magnification (WinRoof version 4.3; Mitani Corporation, Tokyo, Japan). Thirty glomeruli were selected randomly from the superficial and intermediate layers of the cortex and juxtamedulla, and the degrees of glomerular sclerosis and hypertrophy in each area were evaluated separately.

PAS-stained renal sections were divided into 2 areas; one was the superficial and intermediate layers of the cortex and the other was juxtamedulla and outer layer of the medulla. Tubular injuries were evaluated on the basis of the histopathological appearance, that is, tubular dilatation with tubular epithelial attenuation, intraluminal cast, and loss of brush border and tubular atrophy [19]. For quantification, the tubular injury area was measured independently and expressed as ratios relative to the entire areas of the superficial and intermediate layers of the cortex and both juxtamedulla and outer layer of the medulla by using an image analyzer (WinRoof).

\section{Renal Immunohistochemical Analysis}

Target antigens in the preprocessed sections were stained using the indirect immunoperoxidase method, as previously described [19]. The tissue specimens fixed in methyl Carnoy's solution were assessed immunohistochemically for macrophages using a mouse monoclonal antibody (ED-1) specific for CD68 (1:100; Abcam, Tokyo, Japan) and for type I and III collagens using goat polyclonal antibodies specific for each type I (1:200; Southern Biotech, Birmingham, AL, USA) and III collagen (1:200; Southern Biotech). For quantification, images of the entire superficial and intermediate layers of the cortex and both juxtamedulla and outer layer of the medulla were captured separately at $\times 100$ magnification. Positive areas were expressed as ratios relative to the entire areas of the superficial and intermediate layers of the cortex and both juxtamedulla and outer layer of the medulla by using an image analyzer (WinRoof). Furthermore, to evaluate the expansion of the mesan- gial matrix, type IV collagen was labeled using a rabbit polyclonal antibody (1:500; Cedarlane Laboratories, Burlington, ON, Canada) in sections fixed in formalin [19].

Thirty glomeruli were analyzed separately in the areas of the superficial and intermediate layers of the cortex and juxtamedulla for each animal. Briefly, areas positively stained for type IV collagen in each glomerulus and the size of each glomerulus were measured with the WinRoof image analyzer and then expressed as ratios relative to the total glomerular areas.

\section{Immunohistochemical Analysis of Muscle Tissues}

The frozen excised soleus and muscle tissues were cut centrally along the width and embedded in Tissue-Tek O.C.T. Compound (Sakura Finetek Japan Co., Ltd., Tokyo, Japan). Continuous frozen sections sliced to a thickness of $10 \mu \mathrm{m}$ were prepared using a cryostat (HM 550; Thermo Fisher Scientific, Rockford, IL, USA). Double-color immunofluorescent staining was performed to evaluate the diameter of each type 1 and $2 \mathrm{~b}$ fiber as described previously [18]. The muscle sections were incubated with the following primary antibodies: antitype I myosin heavy chain (MyHC) isoform IgG2b (MyHCI; 1:200; BA-F8; Developmental Studies Hybridoma Bank [DSHB], The University of Iowa, Iowa City, IA, USA), antitype IIb MyHC isoform immunoglobulin (Ig)G1 (MyHCIIb; $1: 100 ; \mathrm{F} 18 ; \mathrm{DSHB})$, and antilaminin IgG2a (1:200; 2E8; DSHB) at $4^{\circ} \mathrm{C}$ overnight. After washing, the sections were incubated with the following fluorescent-labeled secondary antibodies at room temperature for $1 \mathrm{~h}$ : Alexa Fluor 488-labeled goat anti-mouse IgG2b (1:500; Invitrogen Corporation, Carlsbad, CA, USA), DyLight 405-labeled goat anti-mouse IgG1 (1:500; Invitrogen Corporation), and Alexa Fluor 488-labeled goat anti-mouse IgG2a (1:500; Invitrogen Corporation). For quantification, the cross-sectional areas of the specimens were evaluated using 300 fibers in 2 visual fields at $\times 100$ magnification per animal by using an image analyzer (WinRoof).

Extraction of Glomerular and Renal Proteins and Western Blot Analysis

The left kidney cortex was separated from the medulla, and glomeruli were isolated with the differential sieving technique using stainless steel grids (80, 150, and 200 mesh sizes) as described previously [19]. Glomerular protein was extracted after homogenization in lysis buffer with protease and phosphatase inhibitors [19]. Next, renal proteins were extracted from portion of the frozen right kidney tissues. Those protein concentrations were measured as previously described [19].

Glomerular protein $(10 \mu \mathrm{g})$ or renal protein extracts $(30 \mu \mathrm{g})$ were separated by sodium dodecyl sulfate-polyacrylamide gel electrophoresis. In glomerular protein, primary antibodies against endothelial nitric oxide synthase (eNOS; rabbit monoclonal; No. 32027; 1:1,000; Cell Signaling Technology, Danvers, MA, USA), phospho-eNOS (Ser1177; rabbit monoclonal; MA5-14957; 1:1,000; Thermo Fisher Scientific), 5'AMP-activated protein kinase (AMPK; rabbit monoclonal; No. 2603; 1:1,000; Cell Signaling Technology), and phospho-AMPK (Thr172; rabbit monoclonal; No. 2535; 1:1,000; Cell Signaling Technology) were incubated overnight at $4^{\circ} \mathrm{C}$. After washes, the membranes were incubated with a horseradish peroxidase-conjugated anti-rabbit antibody (Santa Cruz Biotechnology, Dallas, TX, USA) diluted at 1:2,000 for phospho-eNOS, eNOS, phosphor-AMPK, and AMPK in Blocking One solution for $1 \mathrm{~h}$ at room temperature. 
Table 2. Time-related changes in body weight, blood glucose, SBP, and food intake

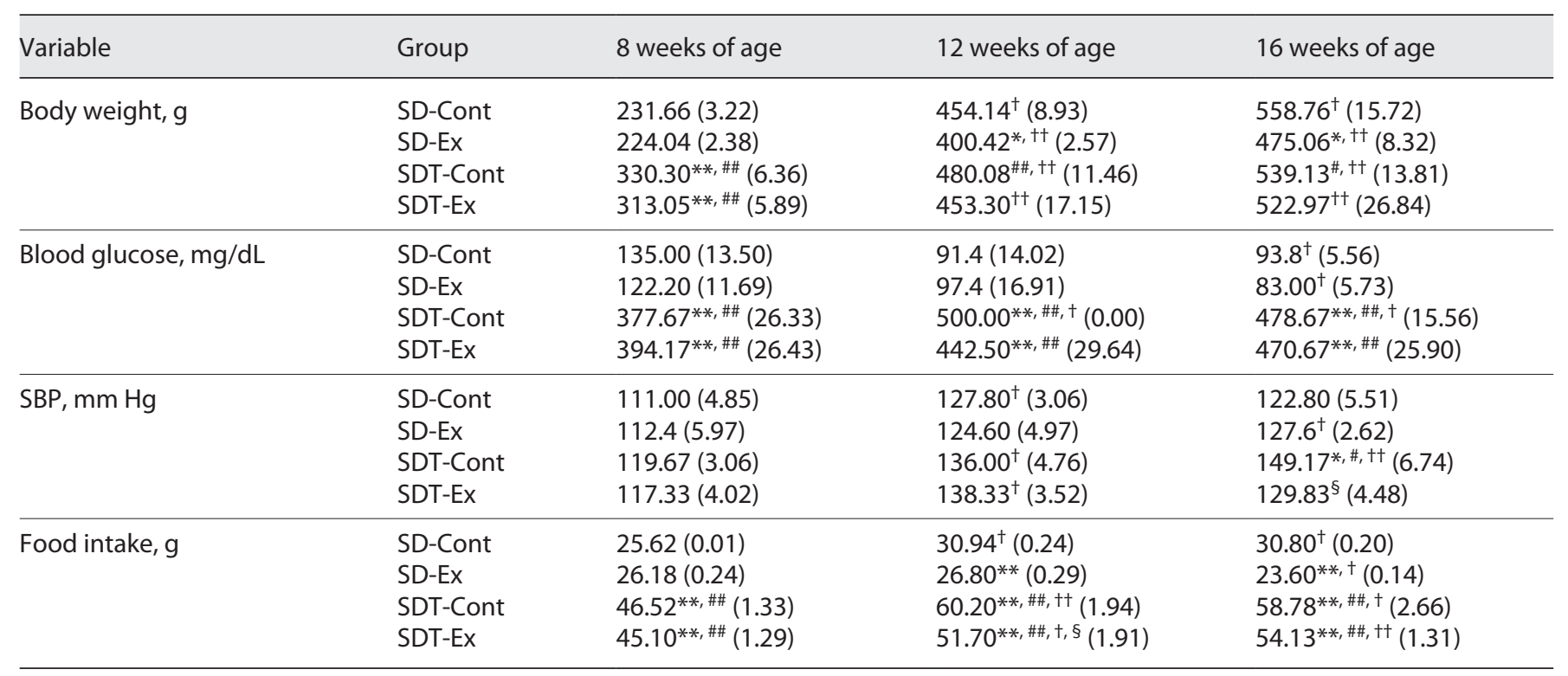

All data were expressed as the mean (SEM). ${ }^{*} p<0.05$ and ${ }^{* *} p<0.01$ versus the SD-Cont group at the same age; ${ }^{\#} p<0.05$ and ${ }^{\# \#} p<$ 0.01 versus the SD-Ex group at the same age; ${ }^{\dagger} p<0.05$ and ${ }^{+\dagger} p<0.01$ versus the same group at age 8 weeks; ${ }^{\S} p<0.05$ versus the SDT-Cont group at the same age. SEM, standard error of the mean.

Regarding the renal protein, primary antibodies against medium-chain acyl-CoA dehydrogenase (MCAD; rabbit monoclonal; ab92461; 1:10,000; Abcam) and proliferator-activated receptor gamma-coactivator-1 alpha (PGC1a; rabbit polyclonal; ab19838; 1:1,000; Abcam, Tokyo, Japan) were used. A rabbit monoclonal antibody to $\alpha$-tubulin $(1: 4,000$, Abcam) was also used to detect a-tubulin on the same membranes. The expression levels of all proteins were quantified using the ImageJ software (the National Institutes of Health, Bethesda, MD, USA). The ratios of phosphorylated protein to total protein and each protein expression level to the $a$-tubulin level were quantitated.

\section{Protein Expression Levels of VEGF in the Glomeruli and}

Kidney

To evaluate the angiogenic activity, both glomerular and renal vascular endothelial growth factor (VEGF) protein expression levels were determined using an ELISA kit (R\&D Systems, Minneapolis, MN, USA) by using protein extracted from the glomerulus and kidney after determining the protein concentrations.

\section{Real-Time Quantitative Reverse Transcription Polymerase}

Chain Reaction

Total RNA was extracted from the kidney tissues using a RNeasy midi kit (Qiagen, Valencia, CA, USA), and TaqMan realtime polymerase chain reaction with StepOnePlus real-time polymerase chain reaction system (Applied Biosystems, Waltham, MA, USA) was used to measure the mRNA levels of hypoxia-inducible factor-1 $\alpha$ (Hif- $1 \alpha$ ) and 18 S ribosomal RNA (18s) in order to evaluate the degree of renal hypoxia. The expression Hif- $1 \alpha$ mRNA levels were normalized to the levels of $18 \mathrm{~s}$ in all the samples.

\section{Evaluation of Oxidative Stress}

To evaluate oxidative stress, urinary 8-hydroxydeoxyguanosine $(8-\mathrm{OHdG})$ levels were measured with high sensitivity using the High Sensitivity 8-OHdG Check ELISA kit (Japan Institute for the Control of Aging, Shizuoka, Japan), and the levels were reported as a daily excretion. Furthermore, real protein carbonyls, which are the most common products of protein oxidation, were quantified using the OxiSelect Protein Carbonyl ELISA Kit (Cell Biolabs, Inc., San Diego, CA, USA) with $10 \mu$ g of protein extracted from the kidney.

\section{Statistical Analysis}

All values were expressed as mean \pm standard error of mean. A $p$ value $<0.05$ was considered statistically significant. After the Kruskal-Wallis test, differences among each group were identified using the Steel test, and the differences among the 4 groups were compared using the Mann-Whitney U test. All statistical analyses were performed using the JMP version 13.0.0 software (SAS Institute, Cary, NC, USA).

\section{Results}

\section{Changes in Body Weight, Blood Glucose Level, SBP,} and Food Intake

The body weight of all the SDT fatty rats was significantly greater than that of all the SD rats at age 8 weeks (Table 2). At ages 12 and 16 weeks, the body weight of all the rats significantly increased compared with that at age 
Table 3. Serum parameters at 16 weeks of age

\begin{tabular}{lllll}
\hline Variable & SD-Cont & SD-Ex & SDT-Cont & SDT-Ex \\
\hline Cystatin C, ng/mL & $4.29(0.12)$ & $4.01(0.30)$ & $5.10(0.19)^{* *, \#}$ & $4.79(0.21)$ \\
Total cholesterol, $\mathrm{mg} / \mathrm{dL}$ & $56.6(3.33)$ & $49.00(1.38)$ & $152.83(7.84)^{* *, \# \#}$ & $120.83(5.95)^{* *, \# \#, \S}$ \\
Triglyceride, $\mathrm{mg} / \mathrm{dL}$ & $52.60(6.53)$ & $41.40(6.71)$ & $401.67(29.46)^{* *, \# \#}$ & $323.67(43.27)^{* *, \# \#}$ \\
Insulin, $\mathrm{ng} / \mathrm{mL}$ & $1.15(0.69)$ & $1.69(0.86)$ & $1.90(0.28)$ & $2.19(0.66)$ \\
IL-6, pg/mL & $83.44(5.42)$ & $72.06(1.89)$ & $67.42(1.74)^{*}$ & $64.33(2.07)^{*, \#}$ \\
BDNF, ng/mL & $11.61(0.61)$ & $11.57(1.41)$ & $28.03(1.15)^{* *, \# \#}$ & $25.41(1.81)^{* *, \# \#}$ \\
\hline
\end{tabular}

All data were expressed as the mean (SEM). ${ }^{*} p<0.05$ and ${ }^{* *} p<0.01$ versus the SD-Cont group; $p<0.05$ and $\# p<0.01$ versus the SD-Ex group; ${ }^{\S} p<0.05$ versus the SDT-Cont group at the same age. SEM, standard error of the mean.

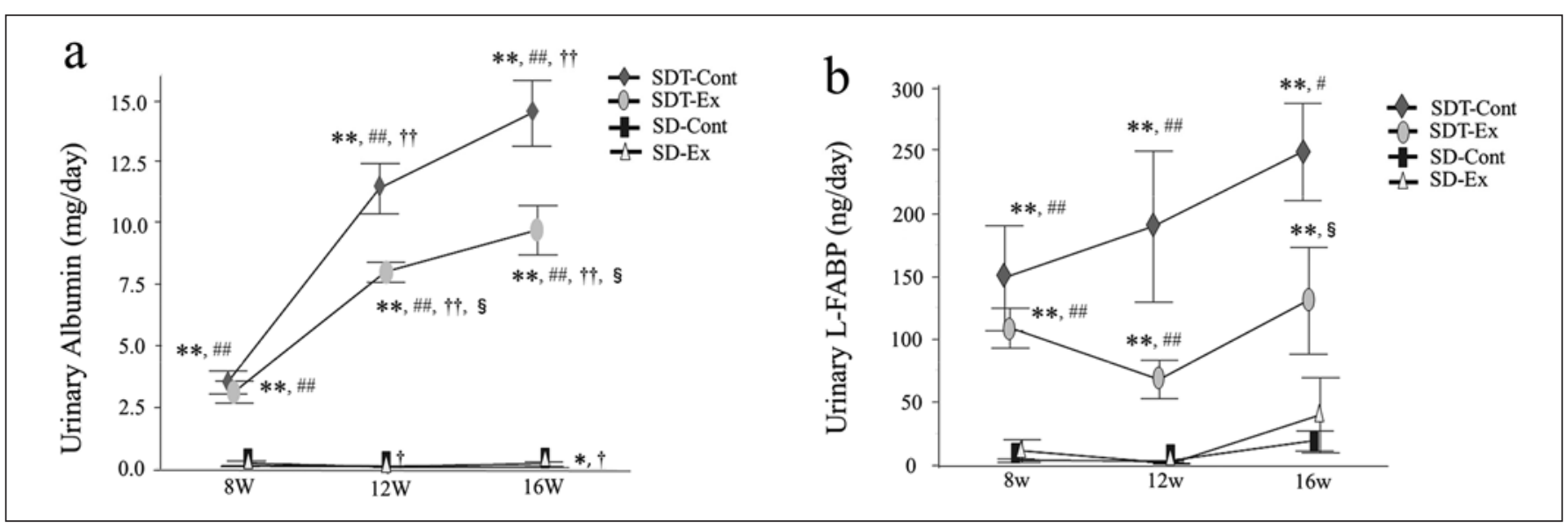

Fig. 1. Changes in urinary parameters of SD (control and exercise) and SDT fatty rats (control and exercise). Urinary albumin levels at 8,12 , and 16 weeks of age (a) and urinary L-FABP levels at 8,12 , and 16 weeks of age (b). SD-Cont group, $n=5$; SD-Ex group, $n=$ 5 ; SDT-Cont group, $n=6$; SDT-Ex group, $n=6$. Values are pre-

8 weeks. While the body weights of SD-Ex group were significantly lower than those of the SD-Cont group at ages 12 and 16 weeks, no significant differences were found between the SDT-Cont and SDT-Ex groups.

Blood glucose levels were significantly higher in the SDT fatty group than in the SD group throughout the experimental period (Table 2). While blood glucose levels in the SD group were significantly lower at age 16 weeks than at age 8 weeks, those in the SDT-Cont group significantly increased at ages 12 and 16 weeks as compared with those at age 8 weeks. The blood glucose levels in SDT-Ex group remained high and were similar to those in the SDT-Cont group.

Although the SBPs were similar in all the rats at ages 8 and 12 weeks, these were significantly higher in the SDT- sented as the mean \pm SEM. ${ }^{* *} p<0.01$ versus the SD group at the same age; ${ }^{\#} p<0.05$ and ${ }^{\# \#} p<0.01$ versus the SD-Ex group at the same age; ${ }^{\dagger \dagger} p<0.01$ versus the same group at 8 weeks of age; ${ }^{\circledR} p<$ 0.05 versus the SDT-Cont group at the same age. SEM, standard error of the mean.

Cont group than in the SD group at age 16 weeks (Table 2). The SBPs significantly increased in the SD-Cont group at age 12 weeks, in the SD-Ex group at age 16 weeks, in the SDT-Cont group at ages 12 and 16 weeks, and in the SDT-Ex group at age 12 weeks as compared with the same group at age 8 weeks. The SBPs were significantly lower in the SDT-Ex group than in the SDT-Cont group at age 16 weeks.

Food intake was significantly higher in the SDT fatty group than in the SD group throughout the experimental period (Table 2). Although food intake in the SD-Ex group significantly decreased at age 16 weeks as compared with age 8 weeks and was significantly lower than that in the SD-Cont group at age 12 and 16 weeks, the food intake in the others significantly increased at ages 12 


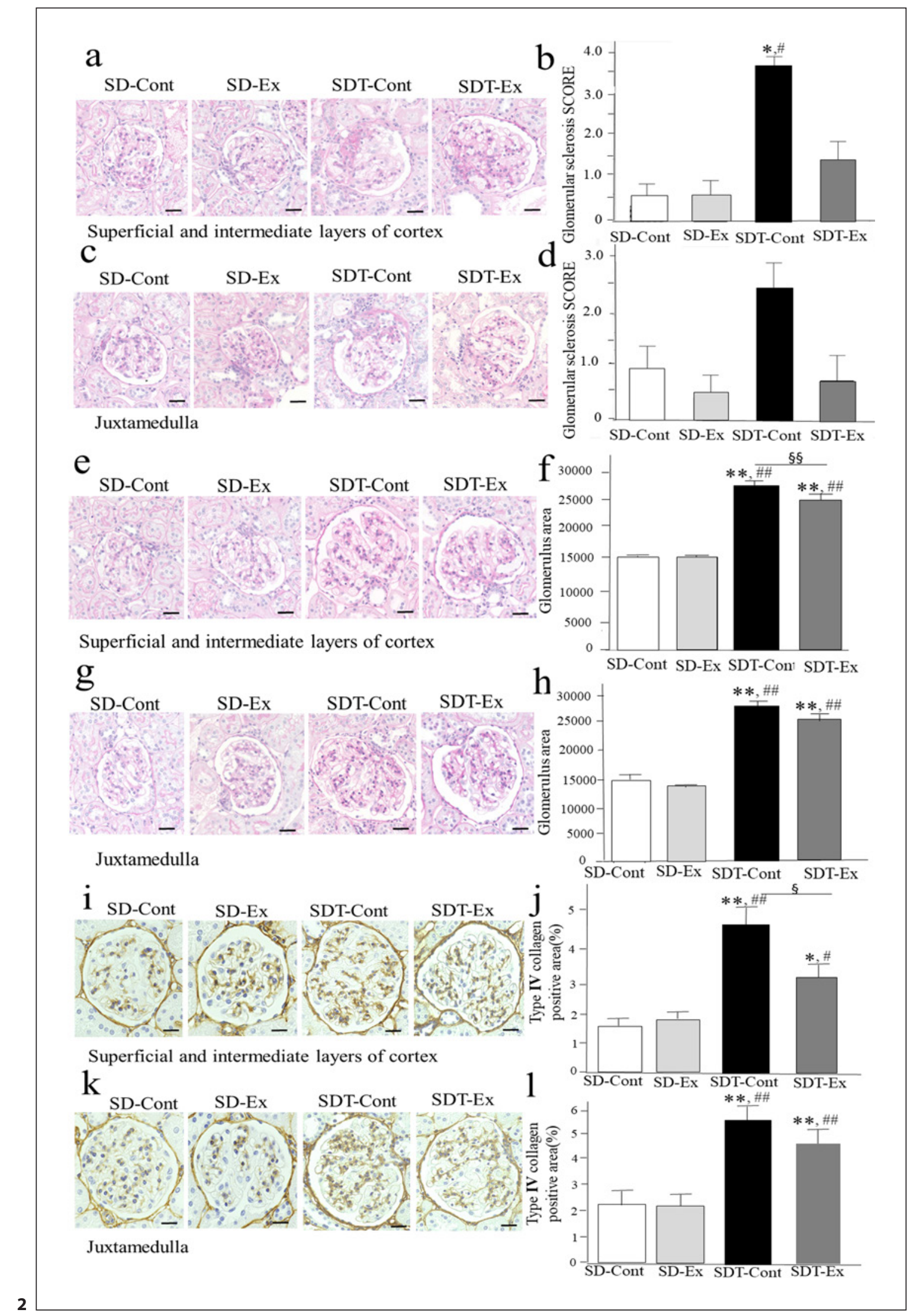

(For legend see next page.) 
and 16 weeks as compared with age 8 weeks. In the SDT fatty rats, food intake was significantly lower in the SDTEx group than in the SDT-Cont group at age 12 weeks.

\section{Comparison of Serum Parameters at Age 16 weeks}

As serum creatinine level is influenced by muscle volume change due to exercise, renal function was evaluated using serum cystatin C level. Serum cystatin C levels were significantly higher in the SDT-Cont group than in the SD group (Table 3), but no significant differences were found between the SD and SDT-Ex groups. Whereas the serum total cholesterol and triglyceride levels were significantly higher in the SDT fatty group than in the SD group, the serum total cholesterol levels were significantly lower in the SDT-Ex group than in the SDT-Cont group (Table 3). Serum insulin levels were slightly higher in the SDT fatty group than in the SD group (Table 3 ). Serum insulin levels were similar between the SDT-Cont and SDT-Ex groups. Serum levels of IL-6 in SDT-Cont group were significantly lower than those in SD-Cont group, and the levels in SDT-Ex group were significantly lower than those in both SD-Cont and SD-Ex groups. Although serum levels of BDNF in all the SDT fatty rats were significantly higher than those in all the SD fats, there were no significant differences between the SDTCont and SDT-Ex groups.

\section{Changes in Urinary Albumin and L-FABP Levels}

To evaluate the degrees of each glomerular and tubular damage using urine, urinary levels of albumin, and LFABP were measured. Urinary albumin levels remained low in the SD group and were significantly decreased in the SD-Ex group at ages 12 and 16 weeks compared with 8 weeks (Fig. 1a). The urinary albumin levels at age 16 weeks were significantly lower in the SD-Ex group than in the SD-Cont group. In all the SDT fatty rats, urinary albumin levels significantly remained higher than in all the SD rats throughout the experimental period, and the levels significantly increased at age 12 and 16 weeks compared with 8 weeks. However, the urinary albumin levels

Fig. 2. Histological staining with PAS showing focal glomerular sclerosis in the superficial and intermediate layers of the cortex (a, b) and juxtamedullary area (c, d). Semiquantitative assessment of focal glomerular sclerosis in the superficial and intermediate layers of the cortex (b) and the juxtamedullary area (d). Histological staining with PAS showing glomerular hypertrophy in the superficial and intermediate layers of the cortex $(\mathbf{e}, \mathbf{f})$ and juxtamedullary area $(\mathbf{g}, \mathbf{h})$. Semiquantitative assessment of glomerulus areas in the superficial and intermediate layers of the cortex (f) and the in the SDT-Ex group at age 12 and 16 weeks were significantly lower than those in SDT-Cont group at the same ages (Fig. 1a).

While the urinary L-FABP levels remained low in the SD group, these remained significantly higher in the SDT-Cont group than in the SD group throughout the experimental period (Fig. 1b). In the SDT-Ex group, urinary L-FABP levels were significantly higher than in the SD group at ages 8 and 12 weeks and were significantly higher than in the SD-Cont group at age 16 weeks (Fig. 1b). The urinary L-FABP levels significantly lower in the SDT-Ex group than in the SDT-Cont group at age 16 weeks (Fig. 1b).

\section{Evaluation of Glomerular Change}

Mild focal glomerular sclerosis in the superficial and intermediate layers of the cortex (Fig. 2a, b) and juxtamedullary area (Fig. 2c, d) was observed in the PASstained kidney tissues from the SDT fatty rats. The glomerular sclerosis scores in the superficial and intermediate layers of the cortex were significantly higher in SDT-Cont group than in the SD group (Fig. 2b), but no significant differences were found between the SD and SDT-Ex groups. The glomerular sclerosis scores in the juxtamedullary area varied widely; therefore, no significant differences were found between the SD and SDT-Ex groups (Fig. 2d).

Glomerular hypertrophy, defined as enlargement of the glomerulus area in PAS-stained kidney tissues (Fig. 2e-h), and type IV collagen immunolocalized to the mesangial areas in the glomeruli (Fig. 2i-l) were significantly observed in the superficial and intermediate layers of the cortex and juxtamedullary area in the SDT fatty rats as compared with the SD rats. In the SDT-Ex group, glomerular hypertrophy (Fig. 2f), and the positive areas of type IV collagen (Fig. $2 \mathrm{j}$ ) in the superficial and intermediate layers of the cortex were significantly suppressed as compared with those in SDT-Cont group, while those in the juxtamedullary area were similar to those in the SDTCont group (Fig. 2h, l).

juxtamedullary area (h). Immunohistological staining using an antibody against type IV collagen in the glomerulus in the superficial and intermediate layers of the cortex $(\mathbf{i}, \mathbf{j})$ and juxtamedullary area $(\mathbf{k}, \mathrm{I})$. SD-Cont group, $n=5$; SD-Ex group, $n=5$; SDT-Cont group, $n=6$; SDT-Ex group, $n=6$. All data were expressed as the mean \pm SEM. ${ }^{*} p<0.05$ and ${ }^{* *} p<0.01$ versus the SD-Cont group, ${ }^{*} p<0.05$ and ${ }^{\# \#} p<0.01$ versus the SD-Ex group, ${ }^{\circledR} p<0.05$ and ${ }^{\circledR s} p<0.01$ versus the SDT-Cont group. Original magnification, $\times 100$. The scale bars represent $20 \mu \mathrm{m}$. SEM, standard error of the mean. 
Fig. 3. Histological staining with PAS showing tubular injury in the superficial and intermediate layers of the cortex $(\mathbf{a}, \mathbf{b})$ and both juxtamedulla and outer layer of the medulla (c, d). Semiquantitative assessment of tubular injury areas in the superficial and intermediate layers of the cortex (b) and both juxtamedulla and outer layer of the medulla (d). Immunohistological staining using antibodies against CD68 (eh), type I collagen (i-I), and type III collagen $(\mathbf{m}-\mathbf{p})$. Original magnification, $\times 100$. SD-Cont group, $n=5$; SD-Ex group, $n=5$; SDT-Cont group, $n=6$; SDT-Ex group, $n$ $=6$. All data were expressed as the mean \pm SEM. ${ }^{*} p<0.05$ and ${ }^{* *} p<0.01$ versus the SD-Cont group, ${ }^{\#} p<0.05$ and ${ }^{\# \#} p<0.01$ versus the SD-Ex group, ${ }^{\$} p<0.05$ versus the SDT-Cont group. Original magnification, $\times 100$. The scale bars represent 100 $\mu \mathrm{m}$. SEM, standard error of the mean.

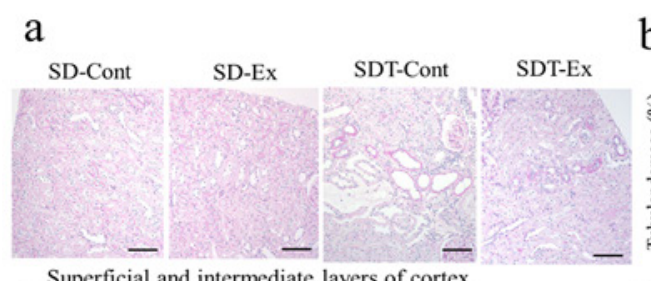

Superficial and intermediate layers of cortex

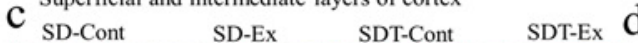
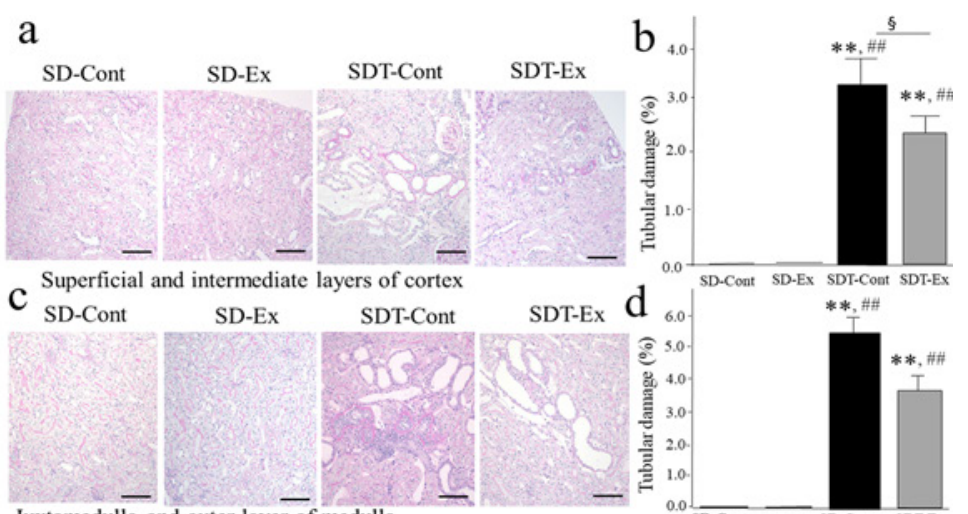

Juxtamedulla and outer layer of medulla<smiles>[CH]</smiles>

SD-Cont SD-Ex

SDT-Cont

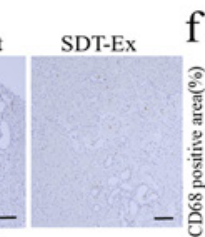

Superficial and intermediate layers of cortex g sD-Cont SD-Ex

SDT-Cont
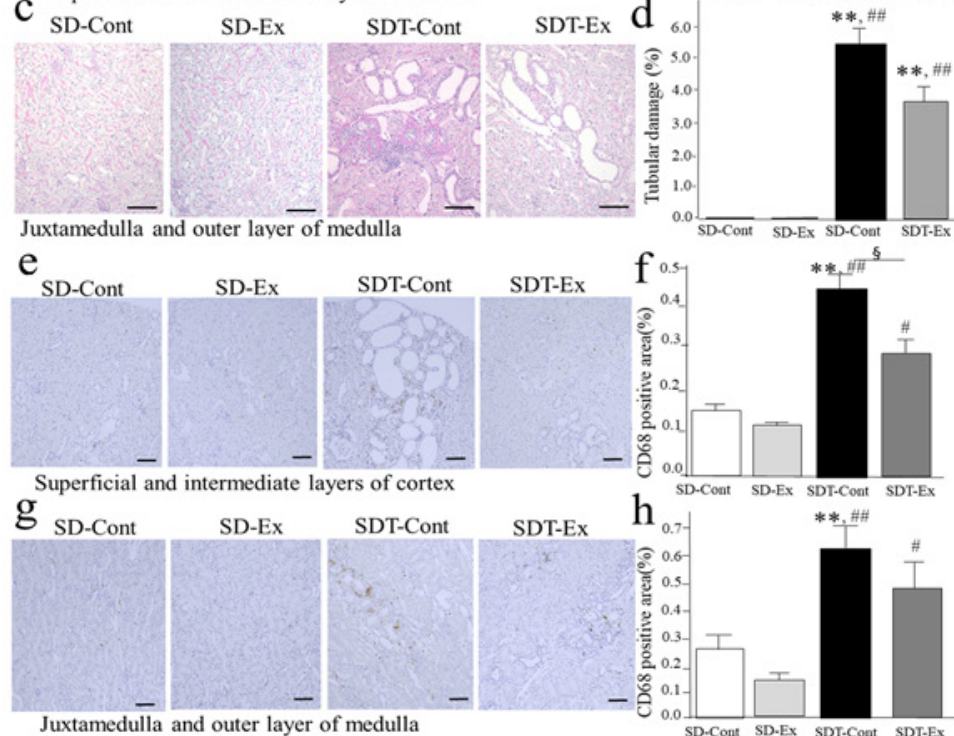

i

SD-Ex

SDT-Cont
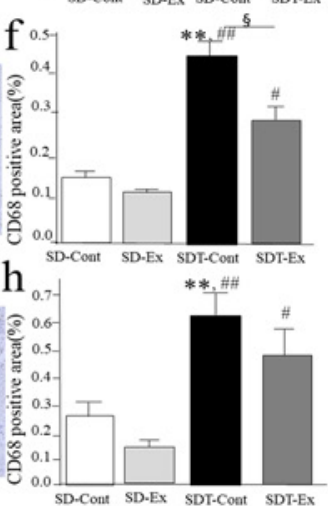

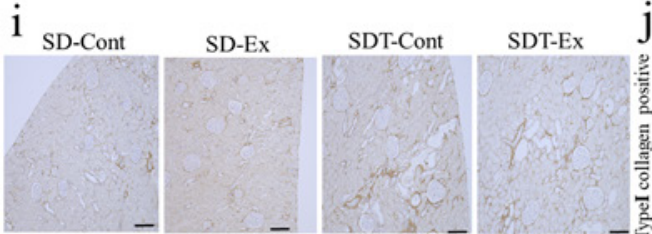

Superficial and intermediate layers of cortex

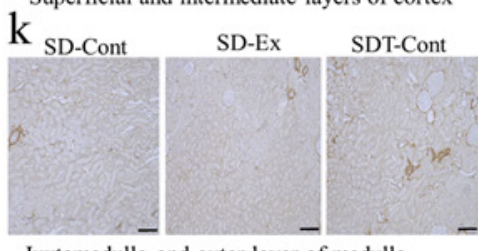

SDT-Ex

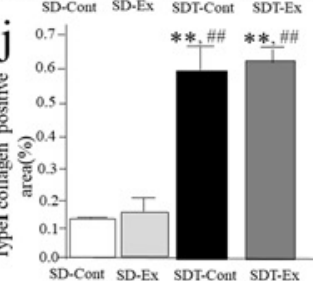

Juxtamedulla and outer layer of medulla

D-Cont SD-Ex SDT-Cont SDT-Ex

$\mathrm{m}$

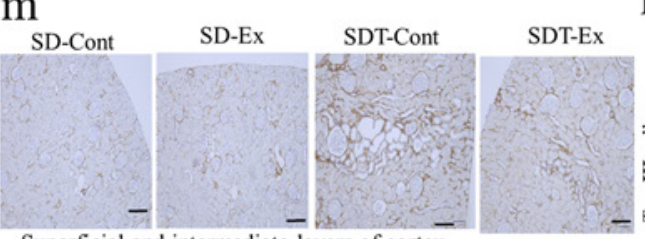

Superficial and intermediate layers of cortex

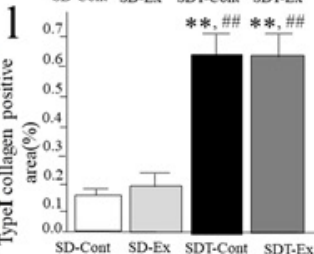

n

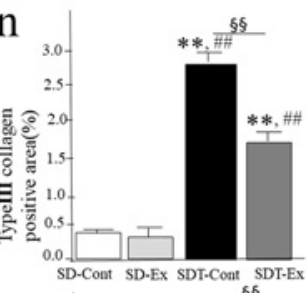

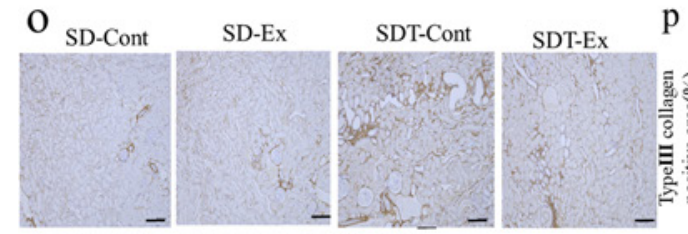

Juxtamedulla and outer layer of medulla

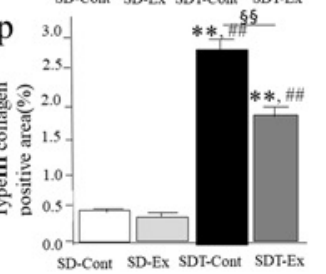


a
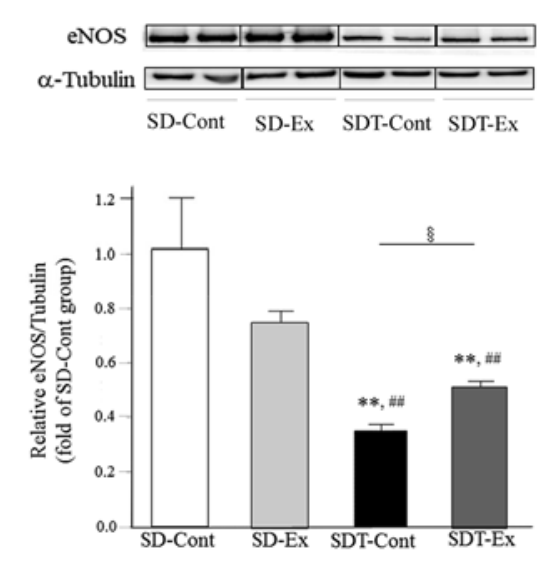

b
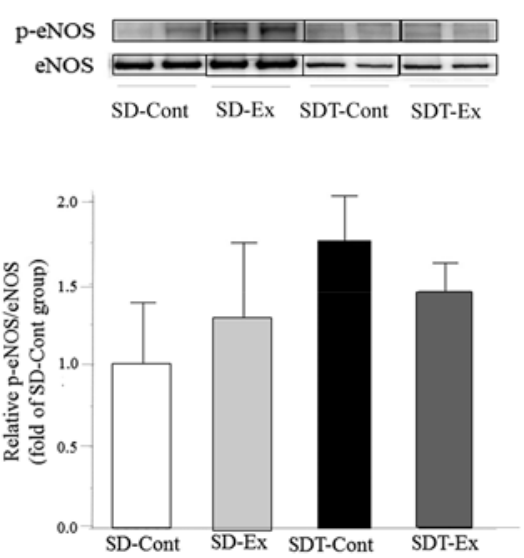

$\mathrm{c}$
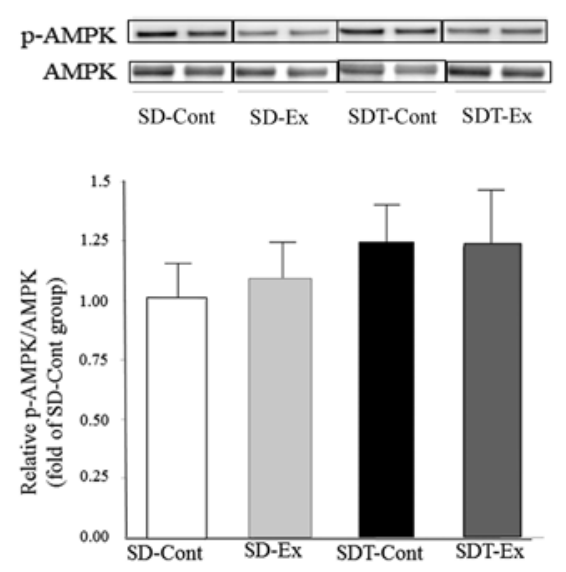

Fig. 4. Glomerular protein in SD and SDT fatty rats, as determined by Western blot analysis. eNOS (a), phosphorylated eNOS (b), and phosphorylated AMPK (c). SD-Cont group, $n=5$; SD-Ex group, $n=5$; SDT-Cont group, $n=6$; SDT-Ex group, $n=6$. All data were

\section{Evaluation of Tubular Damage, Interstitial} Inflammation, and Fibrosis

In the PAS-stained kidney tissues, the degrees of tubular damage in the superficial and intermediate layers of the cortex and juxtamedulla, and outer layer of the medulla were significantly greater in the SDT fatty group than in the SD group (Fig. 3a-d). In the SDT-Ex group, the tubular damages in the superficial and intermediate layers of the cortex were significantly ameliorated as compared with those in the SDT-Cont group, but not those of the juxtamedulla and outer layer of the medulla (Fig. 3b, d).

To evaluate interstitial inflammation, the degree of infiltrated macrophages was quantified. In the superficial and intermediate layers of the cortex and both juxtamedulla and outer layer of the medulla, greater infiltrated macrophages were observed in all the SDT fatty rats than in the SD rats. While the rate of macrophage infiltration in the interstitium of the superficial and intermediate layers of the cortex was significantly lower in the SDT-Ex group than in the SDT-Cont group (Fig. 3e, f), those in the interstitium of the juxtamedulla and outer layer of the medulla were similar between the SDT-Cont and SDT-Ex groups (Fig. 3g, h).

To evaluate interstitial fibrosis, the expressions of type I and III collagen were quantified. The positive areas of type I (Fig. 3i-l) and III collagen (Fig. 3m-p) in the interstitium of the SDT fatty rats were significantly greater than those in the superficial and intermediate

Endurance Exercise Training and Diabetic Kidney Disease expressed as the mean \pm SEM. ${ }^{* *} p<0.01$ versus the SD-Cont group, ${ }^{\# \#} p<0.01$ versus the SD-Ex group, ${ }^{\$} p<0.05$ versus the SDTCont group.

layers of the cortex and both juxtamedulla and outer layer of the medulla of the SD rats. While no significant differences in the type I collagen positive areas were found between the SDT-Cont and SDT-Ex groups (Fig. 3j, 1), the positive areas of type III collagen were significantly smaller in the SDT-Ex group than in the SDT-Cont group (Fig. 3n, p).

\section{Evaluation of Proteins Related to Endothelial Abnormality in the Glomerulus}

To evaluate endothelial abnormalities in isolated glomeruli, the expression levels of eNOS, phosphorylated eNOS, and phosphorylated AMPK related to eNOS phosphorylation were measured. In the glomerulus, the protein expression levels of eNOS were significantly higher in the SDT-Ex group than in the SDT-Cont group, although those in all the SDT fatty group were significantly lower than those in the SD group (Fig. 4a). The phosphorylation levels of eNOS (Fig. 4b) and AMPK (Fig. 4c) were comparable between the groups.

Full-length blots of Western blotting are shown in online supplementary resource Figure S2 (for all online suppl. material, see www.karger.com/doi/10.1159/000521464).

\section{Evaluation of Fatty Acid Metabolism in the Kidney}

To evaluate fatty acid metabolism in the kidney, the protein expression levels of MCAD (Fig. 5a) and PGC1a (Fig. $5 b$ ), which were related to fatty acid $\beta$-oxidation, 


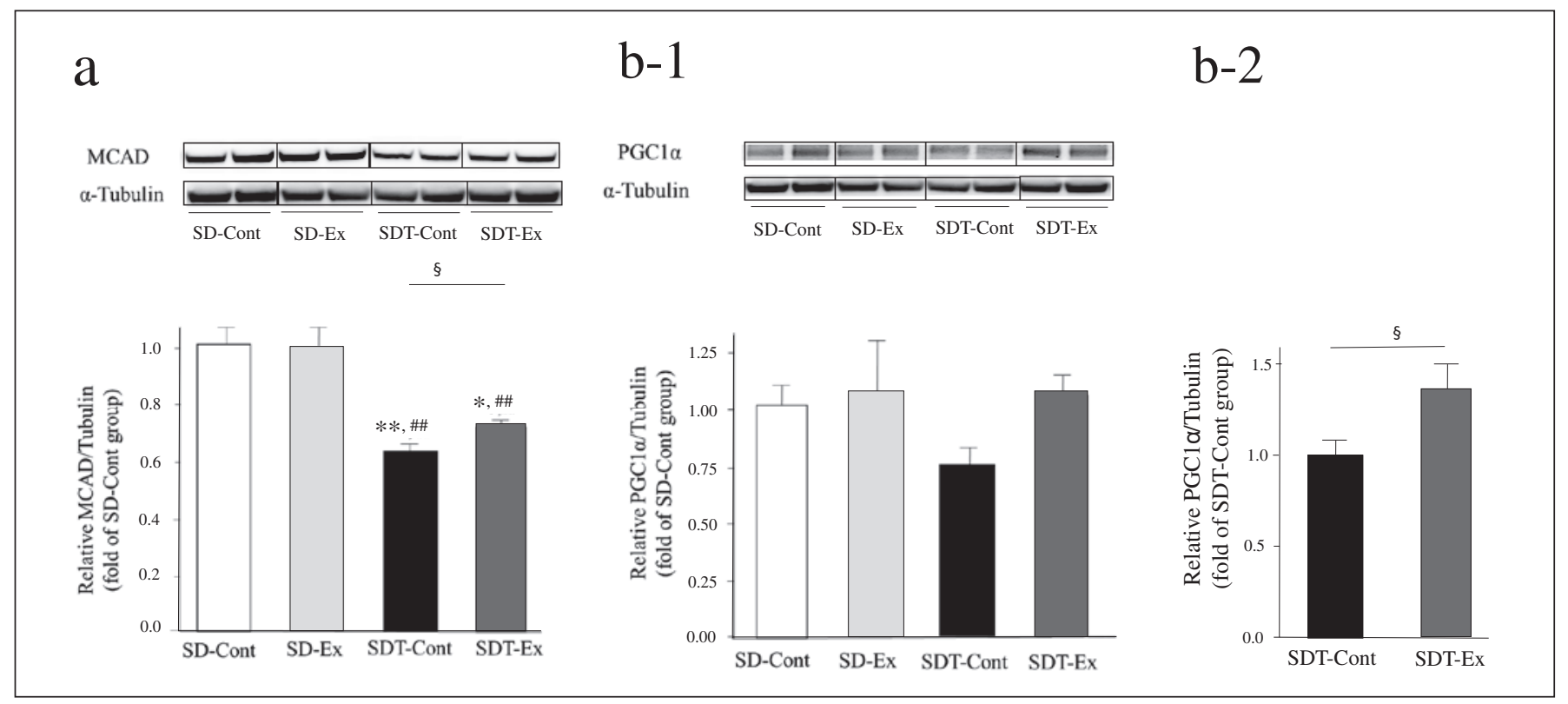

Fig. 5. Renal protein in SD and SDT fatty rats, as determined by Western blot analysis. MCAD (a) and PGC1a (b-1, b-2). SD-Cont group, $n=5$; SD-Ex group, $n=5$; SDT-Cont group, $n=6$; SDT-Ex group, $n=6$. All data were expressed as the mean \pm SEM. ${ }^{*} p<0.05$ and ${ }^{* *} p<0.01$ versus the SD-Cont group, ${ }^{\# \#} p<0.01$ versus the SDEx group, ${ }^{\S} p<0.05$ versus the SDT-Cont group. SEM, standard error of the mean.

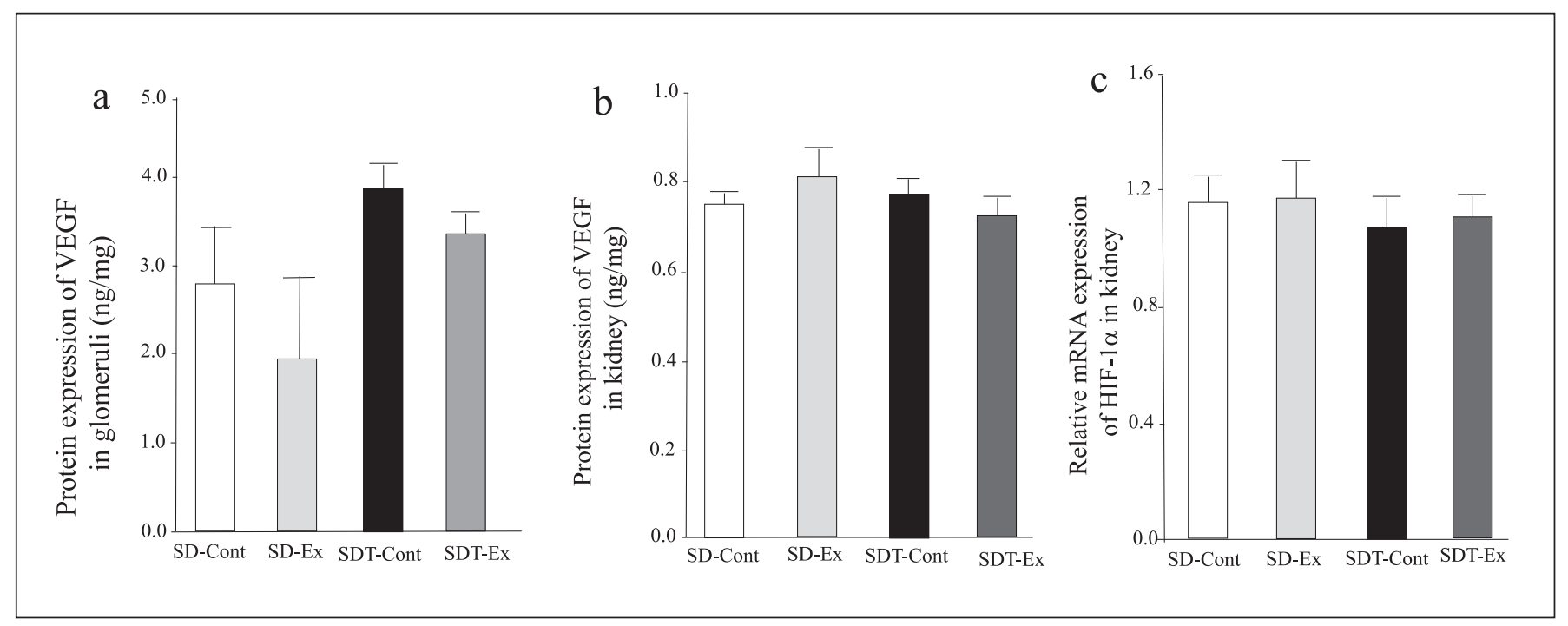

Fig. 6. Evaluation of VEGF protein expression in the glomerulus (a) and kidney (b) and HIF-1a gene expression in the kidney (c). SD-Cont group, $n=5$; SD-Ex group, $n=5$; SDT-Cont group, $n=6$; SDT-Ex group, $n=6$. All data were expressed as the mean \pm SEM. SEM, standard error of the mean.

were measured. The protein expression level of MCAD was significantly lower in the SDT fatty group than in the SD group, but was significantly higher in the SDT-Ex group than in the SDT-Cont group (Fig. 5a). Although no significant differences were found between the groups
(Fig. 5b-1), the protein expression level of PGC1a was significantly higher in the SDT-Ex group than in the SDT-Cont group only in the Mann-Whitney $U$ test (Fig. 5b-2). Full-length blots of Western blotting are shown in online supplementary resource Figure S2. 
a

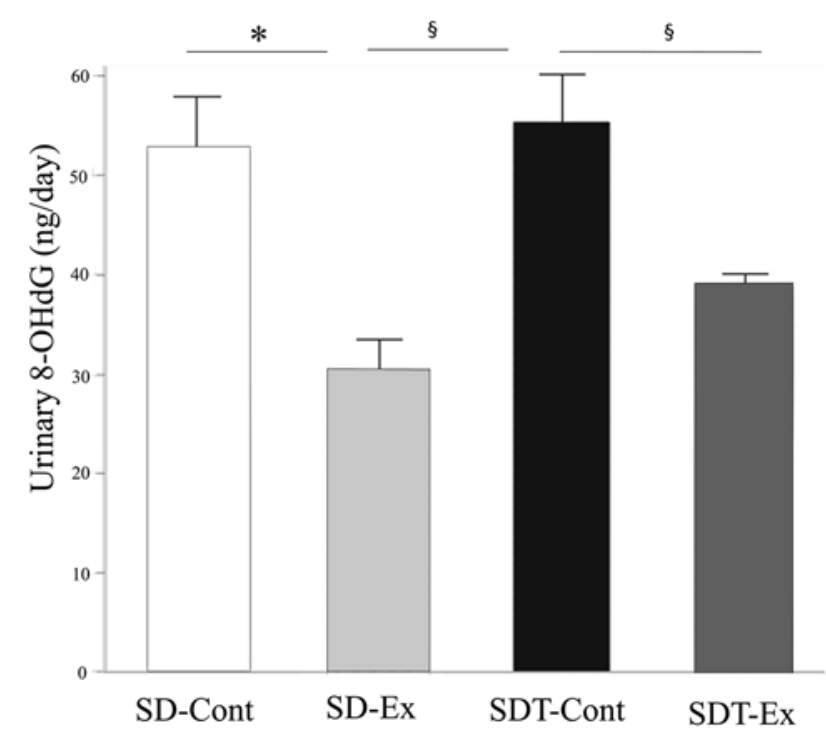

b

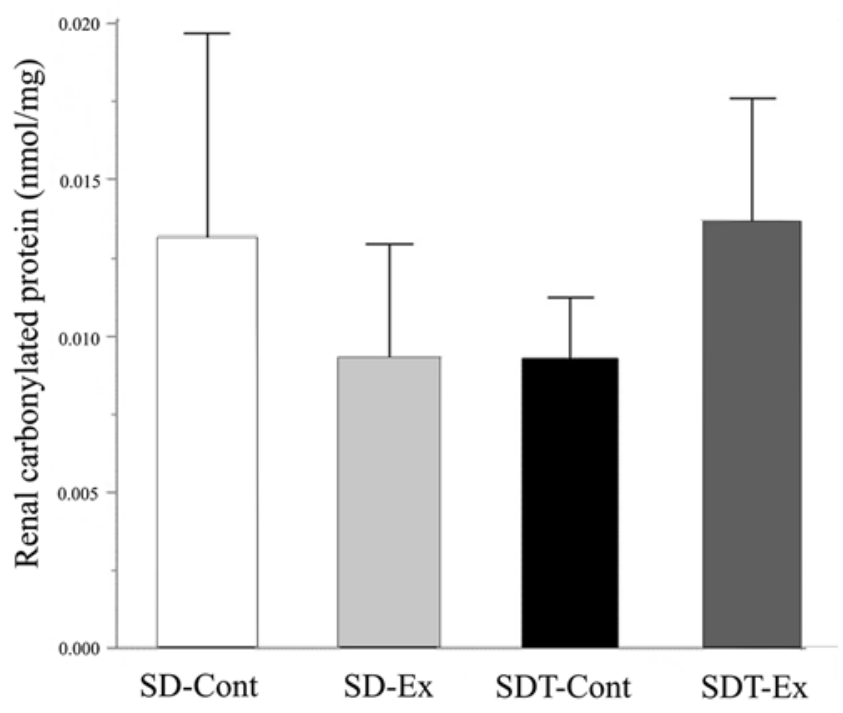

Fig. 7. Urinary 8-OHdG levels as daily excretion in SD and SDT fatty rats at 16 weeks of age (a) and protein carbonyls in the kidney of SD and SDT fatty rats at 16 weeks of age (b). SD-Cont group, $n$ $=5$; SD-Ex group, $n=5$; SDT-Cont group, $n=6$; SDT-Ex group,

\section{Evaluation of VEGF Protein Expression in the}

Glomerulus and Kidney and HIF-1 $\alpha$ Gene Expression in the Kidney

To evaluate the angiogenic activity and the degree of renal hypoxia, the expressions of VEGF and HIF-1 $\alpha$ were measured. The protein expression levels of VEGF in the glomerulus (Fig. 6a) and kidney (Fig. 6b) and the gene expression levels of HIF-1 $\alpha$ in the kidney (Fig. 6c) were not significantly different between the groups.

\section{Evaluation of Oxidative Stress at Age 16 weeks}

To evaluate systemic and renal oxidative stresses, urinary 8 -OHdG and renal protein carbonyls were measured. The urinary 8-OHdG levels were significantly lower in the SD-Ex group than in the SD-Cont and SDT-Cont groups, and in the SDT-Ex group than in the SDT-Cont group (Fig. 7a). However, these were similar between the SD-Cont and SDT-Cont groups. The renal protein carbonyl levels showed no significant differences between all the groups (Fig. 7b). $n=6$. All data were expressed as the mean \pm SEM. ${ }^{*} p<0.05$ versus the SD-Cont group, ${ }^{s} p<0.05$ versus the SDT-Cont group. SEM, standard error of the mean.

Changes in Muscle Strength, Comparison of Muscle

Weight, and Immunohistological Analysis of Muscle

Tissues

The mean muscle strength was lower in the SDT fatty group than in the SD group, but not significantly at ages 8 weeks (Fig. 8a). Although no significant changes were found throughout the experimental period in each group, the muscle strength was significantly lower in the SDTCont group than in the SD group at ages 12 and 16 weeks. In the SDT-Ex group, muscle strength was significantly greater than in the SDT-Cont group at ages 12 and 16 weeks, although it was significantly lower than in SD-Ex group at age 16 weeks (Fig. 8a).

The muscle weights of the soleus (Fig. 8b) and EDL muscles (Fig. 8c) were significantly lower in the SDT fatty group than in the SD group. Although the muscle weight of the EDL muscle was significantly greater in the SDT-Ex group than in the SDT-Cont group, the difference was slight (Fig. 8c).

While the soleus muscle is mainly composed of slowtwitch muscle fibers [22] and a cross-sectional area of type I muscle fibers was evaluated in the soleus (Fig. 8d, 
a

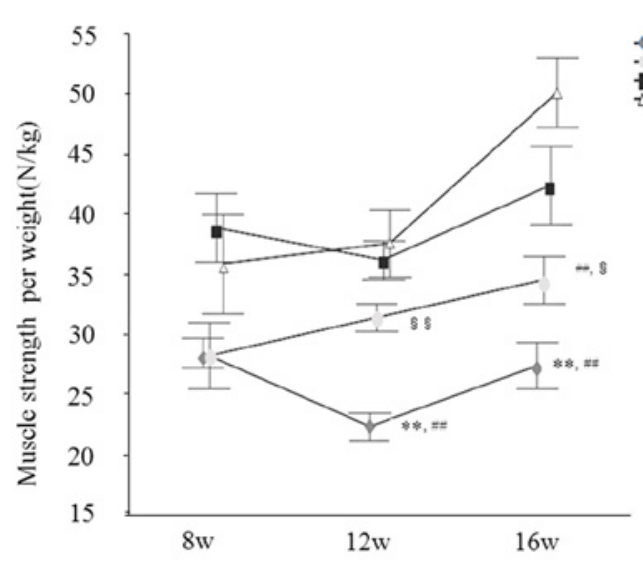

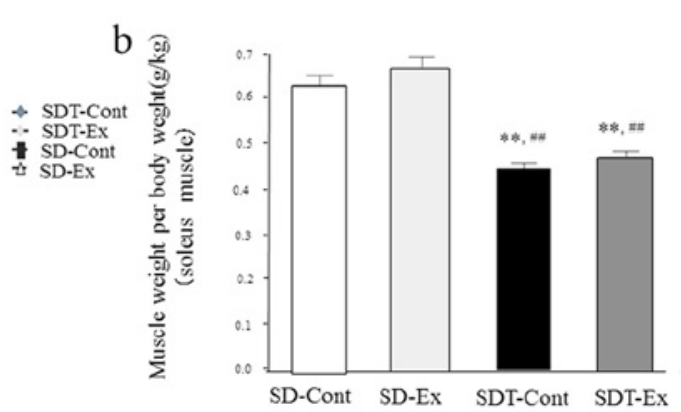

$\mathrm{c}$

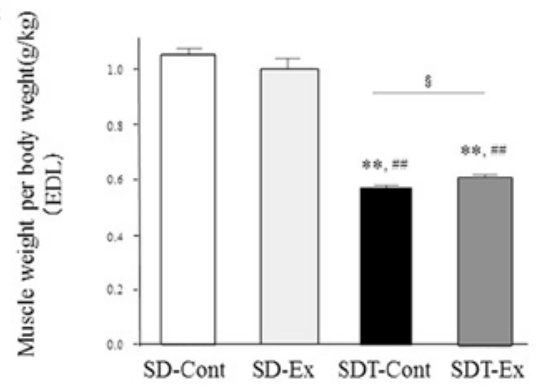

d
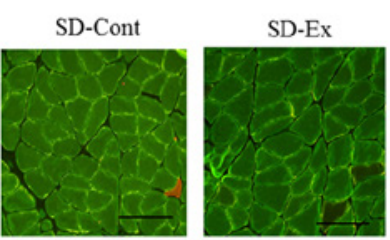

f

SD-Cont

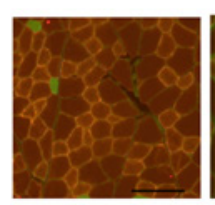

SD-Ex

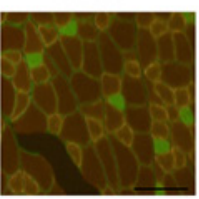

SDT-Cont
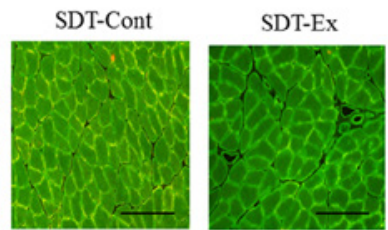

e

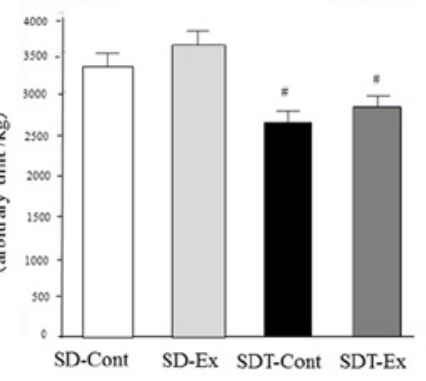

g

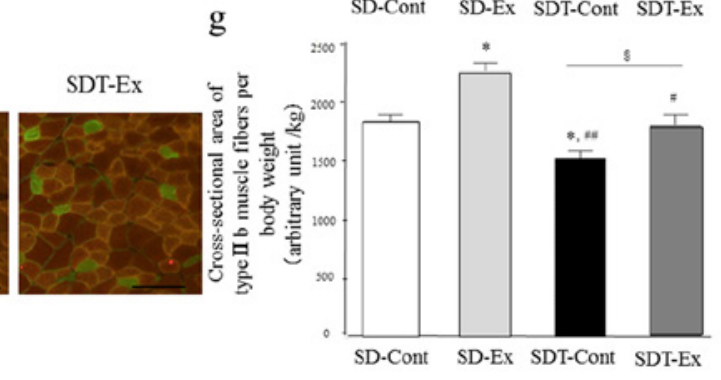

Fig. 8. Evaluation of muscle strength, muscle weight, and muscle histological analysis. a Change in muscle strength per body weight in SD (Control and Exercise) and SDT fatty rats (Control and Exercise). Weight of lower limb muscles per body weight in SD (Control and Exercise) and SDT fatty rats (Control and Exercise). Soleus muscle (b) and EDL muscle (c) at 16 weeks of age. Multiple fluorescent staining of soleus muscle fibers (d) and semiquantitative assessment of type I fibers in the soleus muscle (e). Multiple fluorescent staining of EDL muscle fibers (f) and semiquantitative assessment of type IIb fibers in EDL muscle $(\mathbf{g})$. Type I fibers stained green using antibodies against MyHCI and type IIb fibers stained red using antibodies against MyHCIIb. The frames of the fibers stained green showed laminin. Original magnification, $\times 100$. SD-Cont group, $n=5$; SD-Ex group, $n=5$; SDT-Cont group, $n=6$; SDT-Ex group, $n=6$. All data were expressed as the mean \pm SEM. ${ }^{*} p<0.05$ and ${ }^{* *} p<0.01$ versus the SD-Cont group; ${ }^{*} p<0.05$ and ${ }^{\# \#} p<0.01$ versus the SD-Ex group; ${ }^{\circledR} p<0.05$ and ${ }^{\circledR \S} p<0.01$ versus the SDT-Cont group at the same age. The scale bars represent $100 \mu \mathrm{m} . \mathrm{SEM}$, standard error of the mean. 
Fig. 9. Renal-protective mechanisms of endurance exercise training in SDT fatty rats with type 2 diabetes, hypertension, and hyperlipidemia. The exercise improved hypertension and hyperlipidemia and upregulated expressions of glomerular eNOS, and tubular MCAD and PGC-1 $\alpha$, leading to suppressions of glomerular and tubulointerstitial damage. The endurance exercise training in type 2 diabetes ameliorates DKD by improving endothelial abnormality and enhancing fatty acid metabolism.

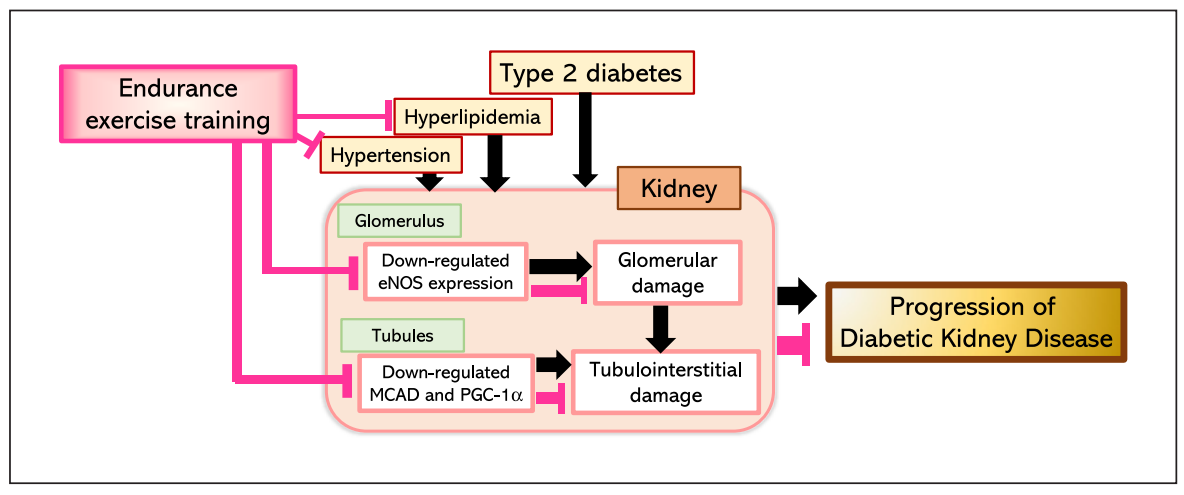

e), the EDL muscle has a high proportion of fast-twitch muscle fibers [22] and a cross-sectional area of type IIb muscle fibers was evaluated in the EDL muscle (Fig. 8f, g). Type I muscle fiber stained green (Fig. 8d) and type IIb muscle fiber stained red (Fig. 8f). The cross-sectional areas of type I muscle fibers in the SDT fatty rats were significantly lower than those in SD-Ex rats, but not in SDCont rats (Fig. 8e). While the cross-sectional areas of type IIb muscle fibers were significantly higher in the SD-Ex group than in the SD-Cont group, these were significantly lower in the SDT-Cont group than in the SD group (Fig. 8g). In SDT-Ex group, although the cross-sectional areas of type IIb muscle fibers were significantly lower than in the SD-Ex group, these were significantly higher than in the SDT-Cont group (Fig. 8g).

\section{Discussion}

The present study revealed that endurance exercise training in SDT fatty rats alleviated hypertension and hyperlipidemia and attenuated the renal morphological damage in the superficial and intermediate layers of the cortex with preventing increases in serum cystatin C, urinary albumin, and L-FABP levels, independently of blood glucose levels. In addition, the exercise increased limb muscle strength and cross-sectional area of the type IIb muscle fiber in EDL muscle. As glomerular eNOS expression and renal both MCAD and PGC-1 $\alpha$ expressions were upregulated by the exercise, prevention of glomerular endothelial abnormality, and acceleration of suppressed renal fatty acid metabolism may be the possible underlying molecular mechanisms of the reno-protective effects of the exercise in the SDT fatty rats (Fig. 9).

Although the body weights in all groups significantly increased during the experimental period as compared with the start of this study, the blood glucose levels at age

Endurance Exercise Training and Diabetic

Kidney Disease
16 weeks decreased in the SD rats as compared with the values at the start. In addition, mild elevation of SBP was observed in the SD rats. However, the levels of each blood glucose and SBP were within normal ranges. Lower body weights and food intakes were observed in the exercise group than in the nonexercise group of SD rats at age 12 and 16 weeks. In the SDT fatty rats, decrease in food intake was induced in the exercise group compared to the nonexercise group at age 12 weeks. As running exercise caused appetite loss by altering appetite-related hormone, ghrelin, and peptide YY levels in healthy males [23], the hormonal changes may be due to the exercise.

The endurance exercise training performed in the present study decreased the SBP and mitigated hyperlipidemia, but not glycemic control in the SDT fatty rats. Although exercise extinguished hyperglycemia and suppressed renal damage in Zucker diabetic rats of another type 2 diabetic animal model [15], the speed, duration, and frequency of the prescribed exercise in the other study were greater than those in our study. Our study revealed that even milder exercise without improvement of glycemic control induced improvements of hypertension and hyperlipidemia and reno-protective effects with prevention of renal dysfunction in type 2 diabetic rats.

The present study showed significant decreases in SBP by endurance exercise training in the SDT fatty rats. Exercise-induced aortic endothelium-dependent vasodilation in type 2 diabetes [24], resulting in a decrease in blood pressure [25]. As hypertension is a risk factor for the progression of DKD, hypotensive action due to the exercise is considered a mechanism by which exercise prevents the progression of DKD. Hypertensive renal damage was reported to occur easily in the juxtamedullary glomeruli and tubulointerstitium downstream from the juxtamedullary glomeruli $[26,27]$. Therefore, we divided the renal section into superficial and intermediate layers of the cortex and juxtamedullary and outer layer of 
the medulla, and evaluated renal morphological changes of the glomerulus and tubulointerstitium in each part separately to confirm the effects of decreased blood pressure. As a result, the beneficial effects of the exercise in the glomerulus were observed in the superficial and intermediate layers of the cortex, but not in the juxtamedulla. The effects in the tubulointerstitium were observed mainly in the superficial and intermediate layers of the cortex, but not in the juxtamedulla and outer layer of the medulla except for collagen type III depositions. These results indicated that other aggravated factors in addition to hypertension might contribute to the onset or progression of renal morphological abnormalities in the juxtamedulla and outer layer of the medulla in the SDT fatty rats at ages 16 weeks. Renal hypoxia is a non-negligible factor for the progression of tubulointerstitial damage. We evaluated the renal VEGF and HIF-1 $\alpha$ expressions, which were upregulated in response to renal ischemia. However, renal upregulations of these molecules were not found in the SDT fatty rats in the present study. The hypotensive effects due to the exercise caused reduction of the collagen type III depositions in the juxtamedulla and outer layer of the medulla, but the effects were limited.

While collagen type III depositions in the interstitium were suppressed by endurance exercise training in the SDT fatty rats, the interstitial collagen type I depositions were not changed. In the SDT fatty rats, the degree of collagen type I depositions in the interstitium was milder than that of collagen type III in the present study. As collagen type I is a mature type of collagen type III, alteration of collagen type I deposition by the exercise might be observed in the kidneys of the SDT fatty rats in longer observational periods.

The lack of eNOS, a major protection of glomerular endothelial cells in type 2 diabetes, provoked diabetic induced-renal histopathologic features [28, 29]. Some studies reported that endurance exercise training produced reno-protective effects by upregulating renal eNOS expression $[7,15]$. Consistent with the previous studies, the present study showed that the exercise upregulated the glomerular eNOS expression suppressed in the SDT fatty rats, although the glomerular endothelial cells showed no apparent morphological abnormalities on transmission electron microscopy in the SDT fatty rats as compared with the SD rats (online suppl. resource Fig. S1) and the phosphorylation levels of eNOS and AMPK were similar between the SD and SDT fatty rats. Therefore, the exercise may attenuate glomerular abnormality in superficial and intermediate layers of the cortex with reduction of urinary albumin level by upregulating glomerular eNOS expression, thereby contributing to prevention of tubulointerstitial damage in superficial and intermediate layers of the cortex with decreased urinary L-FABP level and finally resulting in renal dysfunction.

Artificial hypertrophy of glycolytic muscle fiber by a genetic engineering technique was reported to contribute to the activation of renal eNOS, which suppressed renal damage [30]. In the present study, endurance exercise training elevated cross-sectional area of glycolytic muscle fiber in the EDL muscle, suggesting a relationship between increased glycolytic muscle fiber and glomerular eNOS expression upregulation. However, the molecular mechanism under which the exercise upregulated eNOS in the glomerulus was not confirmed in the present study. Although VEGF secreted from podocyte was important for maintain glomerular endothelial cells [31], VEGF expression in the glomerulus was not increased by the exercise in the present study. Further study is needed to disclose the molecular mechanism.

Recent reports indicated that tubular lipid metabolism disorder caused tubulointerstitial damage by decreasing fatty acid $\beta$-oxidation [32] and that endurance exercise training exerted reno-protection by enhancing the fatty acid metabolism through upregulations of the expressions of renal fatty acid $\beta$-oxidation enzymes and their regulatory factors in hyperlipidemic rats [33]. These findings were supported by the present study showing improvements of hyperlipidemia and upregulations of MCAD and PGC1 $\alpha$ in the SDT fatty rats. The exercise may prevent tubulointerstitial damage via the recovery of fatty acid metabolism in addition to the secondary effects accompanied by decreased urinary albumin level with amelioration of glomerular change in the superficial and intermediate layers of the cortex.

Previous studies focused on the antioxidative effects of endurance exercise training [7, 12], which was supported by the decreased urinary levels of the oxidative marker, 8-OHdG, in the exercise groups of SD and SDT fatty rats in our study. However, we did not show that increased oxidative stress was related to renal damage. While exercise may be useful for reducing systemic oxidative stress, its beneficial effect against DKD may be independent of the antioxidative effects in the SDT fatty rats.

Recent research suggested that skeletal muscle exerted beneficial effects on other organs by secreting myokines, which are physiologically active substances secreted from the muscle fibers [34]. As the present 
study showed that endurance exercise training increased the cross-sectional area of glycolytic muscle fiber, we speculated that myokines produced from the EDL by the exercise partially contributed to the attenuation of renal injury. Although we examined serum IL6 , which is widely known to be elevated by the exercise [34], and serum BDNF, which is reported to have resistance against glomerular injury [35], we found no significant differences between the exercise and nonexercise groups. On the basis of the increase in glycolytic muscle fiber area, which was enhanced by the exercise, in the present study, the production of myokines might not be sufficient for retarding the progression of DKD in type 2 diabetes.

In conclusion, endurance exercise training exerts reno-protective effects by preventing glomerular endothelial abnormality, enhancing renal fatty acid metabolism, and suppressing hypertension and hyperlipidemia in addition to improvement of muscle weakness in type 2 diabetes with obesity, hypertension, and hyperlipidemia, independently of blood glucose. This study proposes a therapeutic potential of regular exercise in DKD in type 2 diabetes.

\section{Acknowledgments}

We thank Ms. Kimie Katayama (Division of Nephrology and Hypertension, Department of Internal Medicine, St. Marianna University School of Medicine), Ms. Junko Asano (Department of Internal Medicine, St. Marianna University School of Medicine), Ms. Naomi Saito, and Ms. Michiyo Ohashi (Department of Anatomy, St. Marianna University School of Medicine), for great technical assistance.

\section{Statement of Ethics}

All these procedures performed in the studies involving animals were in accordance with the ethical standards of the St. Marianna University School of Medicine institution or practice at which the studies were conducted (Approval number: 2008012). All authors approved the final version of the manuscript for publication.

\section{Conflict of Interest Statement}

T. Sugaya is the director and senior scientist of Timewell Medical Co., Ltd., and K. Ohata is the scientist of CMIC HOLDINGS Co., Ltd., the company that produced the kits for L-FABP analysis. None of the other authors have conflicts of interest or financial disclosures of any relevance to the present study.

\section{Funding Sources}

This study was partly supported by JSPS KAKENHI Grant No. JP 20K19554, the Nakatomi Foundation and the Scholarship Fund for Young Researchers, funded by the Promotion and Mutual Aid Corporation for Private Schools of Japan.

\section{Author Contributions}

Study design: H.K., S.Y., Y.O., S.W., K.I., D.I., T.S., K.O., Y.S., and A.K.-I.; data collection: H.K., S.Y., Y.O., S.W., K.I., D.I., Y.N., S.H., and A.K.-I.; data analysis: H.K. and A.K.-I.; data interpretation: H.K., S.Y., Y.O., T.S., K.O., M.W., K.K., Y.S., and A.K.-I.; literature search and figure generation: H.K., K.O., and A.K.-I.; and writing of the manuscript: H.K., Y.O., and A.K.-I.

\section{Data Availability Statement}

All data used to support the findings of this study are available for consultation and for the public, upon request to the corresponding authors (A.K.-I.).

\section{References}

1 Afkarian M, Zelnick LR, Hall YN, Heagerty PJ, Tuttle K, Weiss NS, et al. Clinical manifestations of kidney disease among US adults with diabetes, 1988-2014. JAMA. 2016:316:602-10.

2 Macisaac RJ, Ekinci EI, Jerums G. Markers of and risk factors for the development and progression of diabetic kidney disease. Am J Kidney Dis. 2014;63:S39-62.

3 Yokoyama H, Sone H, Oishi M, Kawai K, Fukumoto Y, Kobayashi M; Japan Diabetes Clinical Data Management Study Group. Prevalence of albuminuria and renal insufficiency and associated clinical factors in type 2 diabetes: the Japan Diabetes Clinical Data Management study (JDDM15). Nephrol Dial Transplant. 2009;24:1212-9.

4 Huang ES, Karter AJ, Danielson KK, Warton EM, Ahmed AT. The association between the

Endurance Exercise Training and Diabetic Kidney Disease number of prescription medications and incident falls in a multi-ethnic population of adult type- 2 diabetes patients: the diabetes and aging study. J Gen Intern Med. 2010;25: 141-6.

5 Namba M, Iwakura T, Nishimura R, Akazawa $\mathrm{K}$, Matsuhisa M, Atsumi Y, et al. The current status of treatment-related severe hypoglycemia in Japanese patients with diabetes mellitus: a report from the committee on a survey of severe hypoglycemia in the Japan Diabetes Society. Diabetol Int. 2018;9:84-99.

6 Odden MC, Peralta CA, Berlowitz DR, Johnson KC, Whittle J, Kitzman DW, et al. Effect of intensive blood pressure control on gait speed and mobility limitation in adults 75 years or older: a randomized clinical trial. JAMA Intern Med. 2017;177:500-7.
7 Agarwal D, Elks CM, Reed SD, Mariappan N Majid DS, Francis J. Chronic exercise preserves renal structure and hemodynamics in spontaneously hypertensive rats. Antioxid Redox Signal. 2012;16:139-52.

8 Baria F, Kamimura MA, Aoike DT, Ammirati A, Rocha ML, de Mello MT, et al. Randomized controlled trial to evaluate the impact of aerobic exercise on visceral fat in overweight chronic kidney disease patients. Nephrol Dial Transplant. 2014;29: 857-64.

9 Kosaki K, Sugaya T, Ohata K, Tanabe J, Hoshino S, Inoue K, et al. Renoprotective effects of voluntary running exercise training on aldosterone-induced renal injury in human L-FABP chromosomal transgenic mice. Hypertens Res. 2019;42:1518-27. 
10 Lazarevic G, Antic S, Vlahovic P, Djordjevic V, Zvezdanovic L, Stefanovic V. Effects of aerobic exercise on microalbuminuria and enzymuria in type 2 diabetic patients. Ren Fail. 2007;29:199-205.

11 Martens CR, Kuczmarski JM, Kim J, Guers JJ, Harris MB, Lennon-Edwards S, et al. Voluntary wheel running augments aortic l-arginine transport and endothelial function in rats with chronic kidney disease. Am J Physiol Renal Physiol. 2014;307:F418-26.

12 Ogawa Y, Takahashi J, Sakuyama A, Xu L, Miura T, Muroya $\mathrm{Y}$, et al. Exercise training delays renal disorders with decreasing oxidative stress and increasing production of 20-hydroxyeicosatetraenoic acid in Dahl salt-sensitive rats. J Hypertens. 2020;38:1336-46.

13 Toyama K, Sugiyama S, Oka H, Sumida H Ogawa H. Exercise therapy correlates with improving renal function through modifying lipid metabolism in patients with cardiovascular disease and chronic kidney disease. J Cardiol. 2010;56:142-6.

14 Amaral LSB, Souza CS, Lima HN, Soares TJ Influence of exercise training on diabetic kidney disease: a brief physiological approach. Exp Biol Med. 2020;245:1142-54.

15 Ito D, Cao P, Kakihana T, Sato E, Suda C, Muroya $\mathrm{Y}$, et al. Chronic running exercise alleviates early progression of nephropathy with upregulation of nitric oxide synthases and suppression of glycation in Zucker diabetic rats. PLoS One. 2015;10:e0138037.

16 Liu $\mathrm{HW}$, Kao HH, Wu CH. Exercise training upregulates SIRT1 to attenuate inflammation and metabolic dysfunction in kidney and liver of diabetic db/db mice. Nutr Metab. 2019;16: 22.

17 Masuyama T, Katsuda Y, Shinohara M. A novel model of obesity-related diabetes: introgression of the Lepr(fa) allele of the Zucker fatty rat into nonobese Spontaneously Diabetic Torii (SDT) rats. Exp Anim. 2005 Jan; 54(1):13-20.
18 Tanabe J, Ogura Y, Kosaki K, Nagai Y, Sugaya $\mathrm{T}$, Ohata K, et al. Relationship between urinary liver-type fatty acid-binding protein (LFABP) and sarcopenia in spontaneously diabetic Torii fatty rats. J Diabetes Res. 2020; 2020:7614035.

19 Yamada S, Tanabe J, Ogura Y, Nagai Y, Sugaya $\mathrm{T}$, Ohata K, et al. Renoprotective effect of GLP-1 receptor agonist, liraglutide, in earlyphase diabetic kidney disease in spontaneously diabetic Torii fatty rats. Clin Exp Nephrol. 2021;25(4):365-75.

20 Lee JC, Kim IY, Son Y, Byeon SK, Yoon DH, Son JS, et al. Evaluation of treadmill exercise effect on muscular lipid profiles of diabetic fatty rats by nanoflow liquid chromatography-tandem mass spectrometry. Sci Rep. 2016;6:29617.

21 Shlipak MG, Matsushita K, Ärnlöv J, Inker LA, Katz R, Polkinghorne KR, et al. Cystatin $\mathrm{C}$ versus creatinine in determining risk based on kidney function. N Engl J Med. 2013 Sep 5;369(10):932-43.

22 Deveci D, Marshall JM, Egginton S. Relationship between capillary angiogenesis, fiber type, and fiber size in chronic systemic hypoxia. Am J Physiol Heart Circ Physiol. 2001;281: H241-52.

23 Broom DR, Batterham RL, King JA, Stensel DJ. Influence of resistance and aerobic exercise on hunger, circulating levels of acylated ghrelin, and peptide YY in healthy males. Am J Physiol Regul Integr Comp Physiol. 2009; 296:R29-35.

24 Sallam N, Khazaei M, Laher I. Effect of moderate-intensity exercise on plasma C-reactive protein and aortic endothelial function in type 2 diabetic mice. Mediators Inflamm. 2010;2010:149678.

25 Schluter KD, Schreckenberg R, da Costa Rebelo RM. Interaction between exercise and hypertension in spontaneously hypertensive rats: a meta-analysis of experimental studies. Hypertens Res. 2010;33:1155-61.

26 Ihara G, Kiyomoto H, Kobori H, Nagai Y, Ohashi N, Hitomi H, et al. Regression of superficial glomerular podocyte injury in type 2 diabetic rats with overt albuminuria: effect of angiotensin II blockade. J Hypertens. 2010;28: 2289-98.
27 Sofue T, Kiyomoto H, Kobori H, Urushihara M, Nishijima Y, Kaifu K, et al. Early treatment with olmesartan prevents juxtamedullary glomerular podocyte injury and the onset of microalbuminuria in type 2 diabetic rats. Am J Hypertens. 2012;25:604-11.

28 Mohan S, Reddick RL, Musi N, Horn DA, Yan B, Prihoda TJ, et al. Diabetic eNOS knockout mice develop distinct macro- and microvascular complications. Lab Invest. 2008;88:51528.

29 Zhao HJ, Wang S, Cheng H, Zhang MZ, Takahashi T, Fogo AB, et al. Endothelial nitric oxide synthase deficiency produces accelerated nephropathy in diabetic mice. J Am Soc Nephrol. 2006;17:2664-9.

30 Hanatani S, Izumiya Y, Araki S, Rokutanda T, Kimura Y, Walsh K, et al. Akt1-mediated fast/ glycolytic skeletal muscle growth attenuates renal damage in experimental kidney disease. J Am Soc Nephrol. 2014;25:2800-11.

31 Eremina V, Cui S, Gerber H, Ferrara N, Haigh J, Nagy A, et al. Vascular endothelial growth factor a signaling in the podocyte-endothelial compartment is required for mesangial cell migration and survival. J Am Soc Nephrol. 2006;17:724-35.

32 Kang HM, Ahn SH, Choi P, Ko YA, Han SH, Chinga $\mathrm{F}$, et al. Defective fatty acid oxidation in renal tubular epithelial cells has a key role in kidney fibrosis development. Nat Med. 2015;21:37-46.

33 Hu G, Xu L, Ma Y, Kohzuki M, Ito O. Chronic exercise provides renal-protective effects with upregulation of fatty acid oxidation in the kidney of high fructose-fed rats. Am Physiol Renal Physiol. 2020;318:F826-34.

34 Pedersen BK, Febbraio MA. Muscles, exercise and obesity: skeletal muscle as a secretory organ. Nat Rev Endocrinol. 2012;8:457-65.

35 Li M, Armelloni S, Zennaro C, Wei C, Corbelli A, Ikehata $\mathrm{M}$, et al. BDNF repairs podocyte damage by microRNA-mediated increase of actin polymerization. J Pathol. 2015; 235:731-44. 